'A E Grulich, II M Poynten, ${ }^{2} \mathrm{~J}$ Roberts, ${ }^{2} \mathrm{~A}$ Farnsworth, ${ }^{3,1} \mathrm{R} \mathrm{J}$ Hillman, ${ }^{1,4} \mathrm{D} \mathrm{J}$ Templeton, ${ }^{5} \mathrm{~S} N$ Tabrizi, ${ }^{5} \mathrm{~S} M \mathrm{M}$ Garland, ${ }^{6} \mathrm{C}$ K Fairley, ${ }^{1} \mathrm{~F}$ Jin. ${ }^{1}$ Kirby Institute, UNSW, Sydney, NSW, Australia; ${ }^{2}$ Douglass Hanly Moir Pathology, Sydney, NSW, Australia; ${ }^{3}$ Western Sydney Sexual Health Centre, University of Sydney, Sydney, NSW, Australia; "RPA Sexual

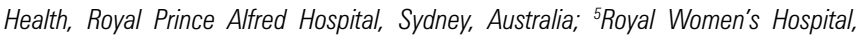
University of Melbourne, Melbourne, Vic, Australia; ${ }^{\circ}$ Melbourne Sexual Health Centre, Melbourne, Vic, Australia

Background Homosexual men have high rates of anal cancer but an understanding of the epidemiology of HSIL, the presumed precursor, is lacking. We aimed to describe the epidemiology of anal HSIL, and association with human papillomavirus (HPV), in a community-recruited cohort of homosexual men.

Methods The Study of the Prevention of Anal Cancer is a threeyear prospective study of anal HPV infection and cancer precursors in homosexual men aged $\geq 35$. At each visit all men receive an anal swab for cytology and HPV genotyping (Roche Linear Array), and high resolution anoscopy with biopsy of suspected lesions. Anal HSIL was defined as having either intraepithelial neoplasia grade $2 / 3$ on histology and/or HSIL on cytology.

Results 293 men were recruited by December 2012. Median age was 49 and $28.3 \%$ were HIV-positive. The baseline prevalence of anal HSIL was $44.6 \%$ and $34.8 \%$ in the HIV-positive and -negative respectively $(p=0.119)$. Among those without HSIL at baseline, HSIL incidence was 30.0 and 20.0 per 100 person-years in the HIVpositive and -negative $(p=0.467)$. Among those with HSIL at baseline, the clearance rate was 37.0 and 44.1 per 100 person-years in the HIV-positive and -negative $(p=0.771)$. Men who tested HPV16 positive on their anal swab at baseline were more likely to develop incident HSIL (58.1 vs 16.1 per 100 person-years, $p=0.002$ ), and less likely to clear prevalent HSIL (15.9 vs. 65.9 per 100 person-years, $\mathrm{p}=0.006$ ).

Conclusion Anal HSIL were highly prevalent. Incidence and clearance were common and closely associated with HPV16 status. The high rate of clearance is consistent with the observation that anal HSIL progress to cancer less commonly than do cervical HSIL. The close association of persistence with HPV16 status suggests that HPV testing should be investigated as a means of identifying those with HSIL who are at highest risk of developing cancer.

\section{P3.136 EPIDEMIC OF LYMPHOGRANULOMA VENEREUM (LGV) IN EUROPE}

doi:10.1136/sextrans-2013-051184.0595

K Haar, G Spiteri, 0 Sfetcu, M van de Laar. European Centre for Disease Prevention and Control (ECDC), Stockholm, Sweden

Background Since 2003, outbreaks of lymphogranuloma venereum (LGV), caused by Chlamydia trachomatis genotypes L1-L3, have been described in men who have sex with men (MSM) in Europe. Awareness increased and enhanced surveillance systems were implemented and several countries in the European Union (EU/ EEA) reported high numbers of LGV cases.

Methods Surveillance for chlamydia, including LGV, in EU/EEA is coordinated by the European Centre for Disease Prevention and Control (ECDC). Since 2009, outbreak information across EU/ EEA is shared via the Epidemic Intelligence Information System for STI (EPIS STI) hosted by ECDC. Data reported by Member States covering the period 2009-2012 were analysed. Individual countries were invited to update detailed information on LGV cases.

Results In 2009-2011, 1642 cases of LGV were reported by 7 Member States: 1630 in men (61\% MSM). Median age was 39 years (range: 18 - 69). Overall, 909 LGV cases (55\%) were HIV-positive. Through EPIS STI, 279 male and 2 female cases from additional 6 countries were reported, mainly in capital areas. Symptoms were reported for 132 cases: 105 cases with and 27 without symptoms.
Proctitis was most commonly reported in men. Among those with known HIV status, $80 \%$ were HIV positive. $45 \%$ of cases in Spain were in migrants and $20 \%$ of the UK cases reported sexual contacts abroad.

Conclusions LGV remains to be primarily found in HIV positive MSM. Asymptomatic presentation hampers case detection and screening and routine genotyping among MSM were suggested to reduce further transmission of LGV. Screening practises differ across countries which results in diversity in number and nature of reported cases. Countries reported individual and clustered LGV cases in EPIS STI with more detailed information than in routine surveillance reports to ECDC. Efforts were made to increase European collaboration to get a better understanding of the evolving LGV epidemic in Europe.

\section{P3.137 RECTAL LUBRICANT USE \& INCIDENT STI INFECTIONS AT 9 US STD CLINICS}

doi:10.1136/sextrans-2013-051184.0596

1.2P M Gorbach, ${ }^{3} \mathrm{D}$ J Feaster, ${ }^{1} \mathrm{H}$ J Pines, ${ }^{3} \mathrm{Z}$ Gomez, ${ }^{4} \mathrm{~J}$ Castro, ${ }^{5} \mathrm{R}$ Bolan, ${ }^{6} \mathrm{~S}$ Henn, ${ }^{7}$ A Douaihy, ${ }^{8} \mathrm{M}$ Golden, ${ }^{3,9} \mathrm{~L}$ Metsch. ${ }^{1}$ University of California, Los Angeles, Los Angeles, CA, United States; 'David Geffen School of Medicine, Los Angeles, CA, United States; ${ }^{3}$ Miller School of Medicine, University of Miami, Miami, FL, United States; ${ }^{4}$ University of Miami, Miami, FL, United States; ${ }^{5}$ Los Angeles Gay and Lesbian Center, Los Angeles, CA, United States; ${ }^{6}$ Whitman-Walker Health, Washington, DC, DC, United States; 'University of Pittsburgh School of Medicine, Pittsburgh, PA, United States, ${ }^{8}$ University of Washington Center for AIDS \& STDs, Seattle, WA, United States; ${ }^{9}$ Columbia University, New York, NY, United States

Background Lubricants (lube) used during receptive anal intercourse (RAI) may affect the acquisition of rectally sexually transmitted infections (rSTIs). We assessed the association between lube use during RAI and rSTI among men who have sex with men (MSM).

Methods In Project Aware, a randomised controlled trial of HIV testing and counselling at 9 US Sexually Transmitted Disease clinics, in 2010 at a 6 month visit 951 MSM completed a web survey on lube use and testing for syphilis and rectal chlamydia and gonorrhoea (rSTIs/syphilis). We used multivariable logistic regression (MVLR) to assess the association of proportion of RAI using lube and rSTIs/syphilis.

Results 589 (61.9\%) of 951 men reported having receptive anal intercourse, of whom $12.9 \%(n=76)$ were diagnosed with $\mathrm{rSTI} /$ syphilis. In the prior month, men reported using lube during a mean of $83 \%$ of RAI acts (median 1.0) and condoms during $54 \%$ (median $67 \%$ ). The mean/median number of unprotected sex partners in the last 6 months (Upartners) was 2.64/1.0. Lube use was not directly associated with $\mathrm{rSTI} /$ syphilis (OR $=0.9995 \% \mathrm{CI}(.60 .1 .66)$. In MVLR adjusting for education, Upartners and condom use, the association between rSTI/syphilis and lube use increased with age and proportion of RAI with lube (interaction $\mathrm{p}=0.02$ ); among men age 32 , the lube use during $50 \%$ and $75 \%$ of RAI acts was associated with adjusted odds ratios of 3.98 (95\% CI 1.07, 14.81) and $5.03(95 \%$ CI 1.01, 25.07), respectively and risk increased at ages greater than 32

Conclusions Although these findings provide further evidence that frequent lubricant use during RAI can facilitate the acquisition of rectal STIs/syphilis among MSM it suggests the risk is only for older MSM. Further research is needed to assess differential risk by types of lubricants across age groups and provide rapid advice to the community who practise RAI on use of safe products.

\section{P3.138 INCREASING ASYMPTOMATIC LYMPHOGRANULOMA VENEREUM INFECTION IN THE UK: RESULTS FROM A NATIONAL CASE-FINDING STUDY}

doi:10.1136/sextrans-2013-051184.0597 